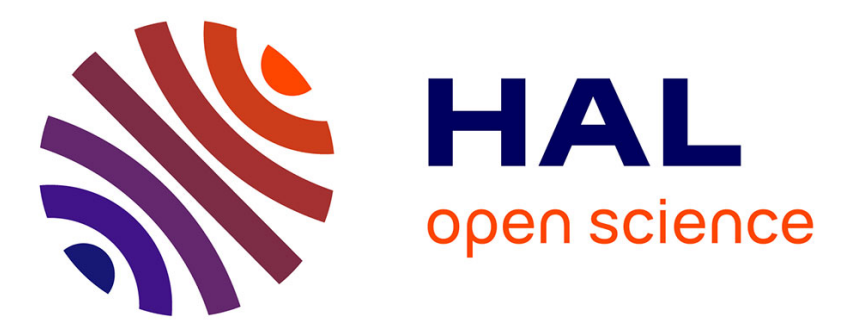

\title{
The vibrational response of a clamped rectangular porous plate
}

Philippe Leclaire, Kirill Horoshenkov, Mark Swift, David Hothersall

\section{To cite this version:}

Philippe Leclaire, Kirill Horoshenkov, Mark Swift, David Hothersall. The vibrational response of a clamped rectangular porous plate. Journal of Sound and Vibration, 2001, 247 (1), pp.19-31. 10.1006/jsvi.2000.3657 . hal-01326043

\section{HAL Id: hal-01326043 https://hal.science/hal-01326043}

Submitted on 6 Jun 2016

HAL is a multi-disciplinary open access archive for the deposit and dissemination of scientific research documents, whether they are published or not. The documents may come from teaching and research institutions in France or abroad, or from public or private research centers.
L'archive ouverte pluridisciplinaire HAL, est destinée au dépôt et à la diffusion de documents scientifiques de niveau recherche, publiés ou non, émanant des établissements d'enseignement et de recherche français ou étrangers, des laboratoires publics ou privés.

\section{(c)(1)}

Distributed under a Creative Commons Attribution| 4.0 International License 


\title{
THE VIBRATIONAL RESPONSE OF A CLAMPED RECTANGULAR POROUS PLATE
}

\author{
P. Leclaire, K.V. Horoshenkov, M.J. Swift and D.C. Hothersall \\ Department of Civil and Environmental Engineering, University of Bradford, Bradford BD7 1 DP, England
}

\begin{abstract}
The vibrations of a rectangular porous plate can be described by two coupled equations involving the time and space derivatives of the deflection and of the relative fluid-solid motion. These equations were established previously and are solved in the present article by Galerkin's variational method. As in the classical theory of plates (non-porous), the solutions are approximate and their mathematical form is chosen. The Rayleigh-Ritz decomposition on the basis of orthogonal or quasi-orthogonal eigenfunctions is used and the solutions are inserted in the vibrational equations. This procedure leads to an explicit form for the solid lateral deflection $w_{s}$ and for the fluid-solid relative diplacement $w$ within the pores. In this study, the chosen eigenfunctions or trial functions are obtained from the linear combination of sinusoidal and hyperbolic functions. The solutions have a single generic form allowing one to account for any boundary condition at the edges involving simply supported, free or clamped edges. Numerical results are given for three different porous plates with the four edges clamped. Simple experiments have also been carried out in order to check the validity of the method. The results are presented and compared with the predictions.
\end{abstract}

\section{INTRODUCTION}

The vibrations of porous structures are important in problems of sound absorption and in the aeronautical industry. The vibration of porous beams and plates has been scarcely studied analytically to date. A recent and significant result has been given by Theodorakopoulos and Beskos [1], who have extended the classical theory of thin rectangular plates to porous materials including Biot's stress-strain relations in porous media [2]. They have established two coupled governing equations and have given the solutions for a simply supported plate by extending Navier's algebraic solution [3] to the porous case. Making an additional assumption about the fluid-solid relative displacement and using an alternate form of Biot's relations [4], Leclaire et al. [5] have proposed two equations of equilibrium, which are valid in applications where the plate is thinner than any acoustic wavelength. The main advantages of the second model are the simplicity of the equations involved without a significant loss of generality, and the possibility to interpret in a more physical manner the fluid-solid interactions associated with the vibration. While the study of boundary conditions other than simply supported from the equations of Theodorakopoulos and Beskos seems to be a difficult task, the equations proposed by Leclaire et al. easily lend themselves to the generalization of the analytical and numerical methods usually applied in the classical theory of plates. An overview of the most common methods can be found in the book by Szilard [3]. The generalization of one of 
these methods is the main subject of this article. In the present study, Galerkin's variational method is applied to porous plates where a classical set of trial functions obtained from the linear combination of trigonometric and hyperbolic functions is chosen. The method can be applied for any boundary condition involving simply supported, clamped or free edges. The particular case of all four edges fully clamped is treated and a comparison, in this configuration, between numerical and experimental results is made for three different porous plates.

Another advantage of the proposed model is that its output parameters are the plate lateral deflection $w_{s}$ and the fluid-solid relative displacement $w$. It can be shown that the simple sum of the time derivatives of $w_{s}$ and $w$ provides the total velocity field in the vicinity of the surface of the plate. The total velocity field is needed in the evaluation of the radiated sound power and the global vibroacoustic indicators. In problems of sound radiation by non-porous plates and structures, recent and refined models include the surface acoustic pressure generated by the plate motion and the effect of the surrounding fluid. The present article is one of the first to study the problem of vibration of porous plates including Biot's equations of relative fluid-solid motion in the pores. Although the fluid-solid interaction is studied, the contribution of the radiation impedance on the solid displacement is neglected in a first approximation. As we are concerned with problems where the fluid is air, the fluid-solid interaction is not symmetrical in the sense that the radiated sound is entirely generated by the solid motion, while the contribution of the fluid on the solid vibration is neglected. This corresponds to the "light" fluid approximation. Obviously, in the case of an acoustical excitation, the plate can be excited acoustically or mechanically, and the fluid does generate the solid vibration. However, this action is included in the excitation term and no other external force due to the radiated acoustic field is taken into consideration. Although this assumption is made, a reasonably good agreement can be found on the plate deflection between the experimental data for different materials saturated by air and the calculated in vacuo response of the plate.

The equations proposed by Leclaire et al. [5] are briefly recalled in section 2. In section 3 , these equations are rewritten in an integral form, as required in Galerkin's variational method, the mathematical form of the solutions is chosen, the coefficients involved in these solutions are calculated from the boundary conditions at the edges and explicit forms are given for $w_{s}$ and $w$. The evaluation of the different elastic coefficients involved in the model is discussed in section 4. Finally, an experimental validation of the theoretical results is proposed in section 5 .

\section{EQUATIONS OF EQUILIBRIUM FOR A POROUS PLATE}

Two simple equations describing the dynamic equilibrium of thin rectangular porous plates have been proposed by Leclaire et al. [5] for a plate of thickness $h$ subjected to a load $q$ and with a pressure difference of $\Delta P=P(-h / 2)-P(+h / 2)$ in the fluid between the two surfaces:

$$
\begin{gathered}
\left(D+\frac{\alpha^{2} M h^{3}}{12}\right) \nabla^{4} w_{s}+h\left(\rho \ddot{w}_{s}+\rho_{f} \ddot{w}\right)=q, \\
\alpha M h \nabla^{2} w_{s}-h\left(\rho_{f} \ddot{w}_{s}+m \ddot{w}\right)=\Delta P .
\end{gathered}
$$

Here $w_{s}$ is the plate deflection, $w$ is the fluid-solid relative displacement, $D$ is the flexural rigidity, $\alpha$ and $M$ are Biot's elastic parameters [6], $\rho$ and $\rho_{f}$ are, respectively, the densities of 
the fluid-solid mixture and of the fluid, $m$ is the mass parameter introduced by Biot [4], given by $\tau_{\infty} \rho_{f} / \phi$, where $\tau_{\infty}$ is the tortuosity and $\phi$ is the porosity. The space derivatives are written with the help of the operators $\nabla^{4}=\nabla^{2}\left(\nabla^{2}\right)$ and $\nabla^{2}=\partial^{2} / \partial x^{2}+\partial^{2} / \partial y^{2}$ of the system of co-ordinates $(x, y)$ while the double dots denote the second time derivative. In the first equation of equilibrium (or plate equation), $\left(D+\alpha^{2} M h^{3} / 12\right) \nabla^{4} w_{s}$ represents the internal potential force (per unit surface) within the fluid-saturated plate, while the inertia terms $h \rho \ddot{w}_{s}$ and $h \rho_{f} \ddot{w}$, and the load $q$ will be considered as external forces. Similarly, an internal force associated with the fluid-solid relative displacement may be defined and is given by $\alpha M h \nabla^{2} w_{s}$, while the external forces can be taken to be $h m \ddot{w}, h \rho_{f} \ddot{w}_{s}$ and $\Delta P$.

\section{VARIATIONAL METHOD FOR SOLVING THE PLATE EQUATIONS}

\subsection{VARIATIONAL EQUATIONS}

According to the virtual work principle, the condition of equilibrium of a system is that the total work performed by the internal and external forces during a small virtual displacement is zero:

$$
\delta \mathbf{W}_{i}+\delta \mathbf{W}_{e}=0
$$

The virtual displacement and the virtual work are defined at constant time. They must be distinguished from the real displacement and from the corresponding work. The virtual work principle can be seen as a particular form of a variational principle formulated as

$$
\int_{z_{1}}^{z_{2}} \delta \mathbf{F ~ d} z=0
$$

According to this principle, a function $\mathbf{F}$ characterizing the evolution of a system from a perturbed state towards a stable equilibrium state is minimum (extremum in a more general manner) between $z_{1}$ and $z_{2}$.

In Galerkin's variational method, the function to be minimized is the total virtual work between the boundaries of the middle surface $(S)$ :

$$
\int_{S} \delta\left(\mathbf{W}_{i}+\mathbf{W}_{e}\right) \mathrm{d} S=0
$$

The virtual work is calculated from a virtual displacement of the system and from forces obtained directly from the governing differential equation which is known a priori and not from the calculation of energies. Upon applying this method to a porous plate and referring to the forces defined in section 2, the internal work in the plate is given by the product of the potential force $\left(D+\alpha^{2} M h^{3} / 12\right) \nabla^{4} w_{s}$ and the virtual displacement $\delta w_{s}$ :

$$
\delta \mathbf{W}_{i}=\left[\left(D+\frac{\alpha^{2} M h^{3}}{12}\right) \nabla^{4} w_{s}\right] \delta w_{s} .
$$

The external work is given by

$$
\delta \mathbf{W}_{e}=\left[h\left(\rho \ddot{w}_{s}+\rho_{f} \ddot{w}\right)-q\right] \delta w_{s} .
$$


Inserting the expressions for $\delta \mathbf{W}_{i}$ and $\delta \mathbf{W}_{e}$ into Equation (5) yields the variational equation of the plate:

$$
\int_{S}\left[\left(D+\frac{\alpha^{2} M h^{3}}{12}\right) \nabla^{4} w_{s}+h\left(\rho \ddot{w}_{s}+\rho_{f} \ddot{w}\right)-q\right] \delta w_{s} \mathrm{~d} S=0 .
$$

For a virtual displacement $\delta w$, a second variational equation can be obtained for the fluid-solid relative displacement by making use of equations (2) and (5):

$$
\int_{S}\left[\alpha M h \nabla^{2} w_{s}-h\left(\rho_{f} \ddot{w}_{s}+\frac{\tau_{\infty}}{\phi} \rho_{f} \ddot{w}\right)-\Delta P\right] \delta w \mathrm{~d} S=0 .
$$

In the following, sinusoidal dependence in time is assumed for all quantities so that the terms containing the time can be discarded, the second derivative introducing the squared angular frequency $\omega^{2}$.

\subsection{CHOICE OF THE MATHEMATICAL FORM OF THE SOLUTIONS}

In the linear domain, the displacements are small and are assumed to be normal to the surface $(a, b)$ defined by the lateral dimensions of the plate which coincide with the system of co-ordinates $(x, y)$. The problem consists in finding suitable expressions for $w_{s}$ and $w$ verifying the system of integral equations (8) and (9) as well as satisfying the boundary conditions at the edges of the plate. Various approximate functions have been tried in the case of non-porous plates where only one governing equation is considered, such as a linear combination of trigonometric and hyperbolic functions [7, 8], polynomials (see reference [9] for example) or trigonometric functions [10]. The following approach is used in the present study: a possible solution for the solid deflection is sought first. The general form of this solution is also applied to the other unknown $w$ and to the excitation terms. All these terms are then inserted in equations (8) and (9) leading to new equations. Finding solutions to these is a verification of their compatibility and provides the amplitude coefficients of $w_{s}$ and $w$.

The form of the trial functions chosen is the combination of trigonometric and hyperbolic functions. As in the Rayleigh-Ritz method, the classical decomposition of the solutions on the basis of orthogonal eigenfunctions is used. The solid deflection is written as

$$
w_{s}(x, y)=\sum_{r=1}^{\infty} \sum_{n=1}^{\infty} W_{r n}^{s} \Phi_{r n}(x, y)
$$

Since the plate is rectangular, it is convenient to write each function $\Phi_{r n}(x, y)$ as the product of two independent functions called the beam function $\theta_{r}(x)$ and $\psi_{n}(y)$ of one variable only:

$$
\Phi_{r n}(x, y)=\theta_{r}(x) \psi_{n}(y)
$$

The chosen form for the beam function is

$$
\theta_{r}(x)=C_{1 r} \sin \left(\lambda_{r} \frac{x}{a}\right)+C_{2 r} \cos \left(\lambda_{r} \frac{x}{a}\right)+C_{3 r} \sinh \left(\lambda_{r} \frac{x}{a}\right)+C_{4 r} \cosh \left(\lambda_{r} \frac{x}{a}\right)
$$

where $\lambda_{r}$ is a frequency parameter corresponding to the rth root of a characteristic equation. The mathematical form of this equation and the coefficients $C_{1 r}, C_{2 r}, C_{3 r}, C_{4 r}$ are imposed by the boundary conditions. 


\subsection{BOUNDARY CONDITIONS}

The standard boundary conditions are applied to the porous solid which is considered as a homogeneous effective medium in Biot's approach of porous media. For a clamped beam of length $a$, the conditions at the edges are that the solid displacement and its first derivative are zero: $w_{s}(0)=w_{s}(a)=0$ and $w_{s}^{\prime}(0)=w_{s}^{\prime}(a)=0$, corresponding to edges without motion of translation or rotation. The determinant of the homogeneous boundary conditions is then obtained from the following equations:

$$
\begin{array}{ccccc}
0 & +C_{2 r} & +0 & +C_{4 r} & =0, \\
\lambda_{r} C_{1 r} & +0 & +\lambda_{r} C_{3 r} & +0 & =0, \\
C_{1 r} \sin \lambda_{r} & +C_{2 r} \cos \lambda_{r} & +C_{3 r} \sinh \lambda_{r} & +C_{4 r} \cosh \lambda_{r} & =0, \\
C_{1 r} \cos \lambda_{r} & -C_{2 r} \sin \lambda_{r} & +C_{3 r} \cosh \lambda_{r} & +C_{4 r} \sinh \lambda_{r} & =0,
\end{array}
$$

which are verified for

$$
C_{4 r}=-C_{2 r}=1, \quad C_{1 r}=-C_{3 r}=\frac{\cosh \left(\lambda_{r}\right)-\cos \left(\lambda_{r}\right)}{\sinh \left(\lambda_{r}\right)-\sin \left(\lambda_{r}\right)}
$$

and for $\lambda_{r}$ satisfying the following characteristic equation or frequency equation:

$$
\cos \left(\lambda_{r}\right) \cosh \left(\lambda_{r}\right)=1 \text {. }
$$

Numerical values for $\lambda_{r}$ can be found in reference [7]. The values of $C_{1 r}, C_{2 r}, C_{3 r}, C_{4 r}$ and $\lambda_{r}$ are then inserted into the expression for the beam function $\theta_{r}(x)$. The beam function in the $y$ direction $\psi_{n}(y)$ is deduced from the expression for $\theta_{r}(x)$, where $x, a$ and $r$ are replaced by $y$, $b$ and $n$.

If the beam is simply supported, $C_{1 r}=1, C_{2 r}=C_{3 r}=C_{4 r}=0$ and the frequency equation is $\sin \left(\lambda_{r}\right)=0$. The same generic expression can be used for $\theta_{r}(x)$ in the case of other boundary conditions involving a combination of simply supported, free, clamped or guided edges. Each case is described by a different set of coefficients $C_{1 r}, C_{2 r}, C_{3 r}, C_{4 r}$ and a frequency equation leading to different values of $\lambda_{r}$.

\subsection{SOLUTIONS}

In this study, one seeks solutions for $w(x, y), \delta w_{s}, \delta w, q$ and $\Delta P$ with the same mathematical form as $w_{s}$ :

$$
\begin{gathered}
w(x, y)=\sum_{r=1}^{\infty} \sum_{n=1}^{\infty} W_{r n} \Phi_{r n}(x, y), \\
\delta w_{s}(x, y)=\sum_{r=1}^{\infty} \sum_{n=1}^{\infty}\left(\delta w_{s}\right)_{r n} \theta_{r}(x) \psi_{n}(y), \\
\delta w(x, y)=\sum_{r=1}^{\infty} \sum_{n=1}^{\infty} \delta w_{r n} \theta_{r}(x) \psi_{n}(y), \\
q(x, y)=\sum_{r=1}^{\infty} \sum_{n=1}^{\infty} Q_{r n} \theta_{r}(x) \psi_{n}(y), \\
\Delta P(x, y)=\sum_{r=1}^{\infty} \sum_{n=1}^{\infty} \Delta P_{r n} \theta_{r}(x) \psi_{n}(y) .
\end{gathered}
$$


Here, the coefficients $W_{r n}^{s}$ and $W_{r n}$ are the unknowns of the problem. After insertion into equations (8) and (9), the solutions, if they exist, must satisfy the following equations:

$$
\begin{gathered}
\sum_{r=1}^{\infty} \sum_{n=1}^{\infty} \int_{0}^{a} \int_{0}^{b}\left(W _ { r n } ^ { s } \left[\left(D+\frac{\alpha^{2} M h^{3}}{12}\right)\left(\theta_{r}^{(\mathrm{IV})} \psi_{n} \theta_{i} \psi_{k}+2 \theta_{r}^{(\mathrm{II})} \psi_{n}^{\prime \prime} \theta_{i} \psi_{k}+\theta_{r} \psi_{n}^{(4)} \theta_{i} \psi_{k}\right)\right.\right. \\
\left.\left.-h \rho \omega^{2} \theta_{r} \psi_{n} \theta_{i} \psi_{k}\right]-W_{r n} \rho_{f} \omega^{2} h \theta_{r} \psi_{n} \theta_{i} \psi_{k}-Q_{r n} \theta_{r} \psi_{n} \theta_{i} \psi_{k}\right) \mathrm{d} x \mathrm{~d} y=0, \\
\sum_{r=1}^{\infty} \sum_{n=1}^{\infty} \int_{0}^{a} \int_{0}^{b}\left(W_{r n}^{s}\left[\alpha M h\left(\theta_{r}^{(\mathrm{II})} \psi_{n} \theta_{i} \psi_{k}+\theta_{r} \psi_{n}^{(\mathrm{II})} \theta_{i} \psi_{k}\right)+h \rho_{f} \omega^{2} \theta_{r} \psi_{n} \theta_{i} \psi_{k}\right]\right. \\
\left.-W_{r n} h \frac{\tau_{\infty}}{\phi} \rho_{f} \omega^{2} \theta_{r} \psi_{n} \theta_{i} \psi_{k}-\Delta P_{r n} \theta_{r} \psi_{n} \theta_{i} \psi_{k}\right) \mathrm{d} x \mathrm{~d} y=0 .
\end{gathered}
$$

The fourth and second order derivatives are, respectively, denoted by the exponents (IV) and (II). The eigenfunctions and their derivatives satisfy the orthogonality requirement (depending on the choice of eigenfunctions, this condition can be satisfied approximately). The terms with non-identical subscripts $r, i$ and $n, k$ are therefore zero or negligible and equations (15) and (16) can be rewritten as

$$
\begin{gathered}
\sum_{r=1}^{\infty} \sum_{n=1}^{\infty}\left(W_{r n}^{s}\left[\left(D+\frac{\alpha^{2} M h^{3}}{12}\right)\left(I_{1} I_{2}+2 I_{3} I_{4}+I_{5} I_{6}\right)-h \rho \omega^{2} I_{2} I_{6}\right]\right. \\
\left.-W_{r n} h \rho_{f} \omega^{2} I_{2} I_{6}\right)=\sum_{r=1}^{\infty} \sum_{n=1}^{\infty} Q_{r n} I_{2} I_{6} . \\
\sum_{r=1}^{\infty} \sum_{n=1}^{\infty}\left(W_{r n}^{s}\left[\alpha M h\left(I_{2} I_{3}+I_{4} I_{6}\right)+h \rho_{f} \omega^{2} I_{2} I_{6}\right]+W_{r n} h m \omega^{2} I_{2} I_{6}\right)=\sum_{r=1}^{\infty} \sum_{n=1}^{\infty} \Delta P_{r n} I_{2} I_{6},
\end{gathered}
$$

where $\left(I_{1}\right)-\left(I_{6}\right)$ are the simple definite integrals

$$
\begin{array}{ll}
I_{1}=\int_{0}^{a} \theta_{r}^{(\mathrm{IV})}(x) \theta_{r}(x) \mathrm{d} x, & I_{5}=\int_{0}^{b} \psi_{n}^{(\mathrm{IV})}(y) \psi_{n}(y) \mathrm{d} y, \\
I_{6}=\int_{0}^{a} \theta_{r}(x) \theta_{r}(x) \mathrm{d} x, & I_{2}=\int_{0}^{b} \psi_{n}(y) \psi_{n}(y) \mathrm{d} y, \\
I_{3}=\int_{0}^{a} \theta_{r}^{(\mathrm{II})}(x) \theta_{r}(x) \mathrm{d} x, & I_{4}=\int_{0}^{b} \psi_{n}^{(\mathrm{II})}(y) \psi_{n}(y) \mathrm{d} y .
\end{array}
$$

Solving system $(17,18)$ for a couple $(r, n)$ yields the coefficients $W_{r n}^{s}, W_{r n}$ and the responses are given by equations (10) and (11). For a simply supported plate, the integrals take a simple form and a solution similar to Navier's algebraic solution [3] for a porous plate [1] is obtained.

The coefficients $Q_{r n}$ and $\Delta P_{r n}$ correspond to the external exciting forces applied to the porous plate and are determined by the way the plate is excited. Rigorously, the external forces are responsible for the motion of the plate, which in turn, contributes to the loading of 
the plate but this contribution is neglected in this study in a first approximation. The coefficients $Q_{r n}$ and $\Delta P_{r n}$ can be written [3] as

$$
\begin{aligned}
Q_{r n} & =\int_{0}^{a} \int_{0}^{b} q(x, y) \theta_{r}(x) \psi_{n}(y) \mathrm{d} x \mathrm{~d} y / \int_{0}^{a} \int_{0}^{b} \theta_{r}^{2}(x) \psi_{n}^{2}(y) \mathrm{d} x \mathrm{~d} y, \\
\Delta P_{r n} & =\int_{0}^{a} \int_{0}^{b} \Delta P(x, y) \theta_{r}(x) \psi_{n}(y) \mathrm{d} x \mathrm{~d} y / \int_{0}^{a} \int_{0}^{b} \theta_{r}^{2}(x) \psi_{n}^{2}(y) \mathrm{d} x \mathrm{~d} y .
\end{aligned}
$$

For a load $F_{0}$ concentrated at one point $\left(x_{0}, y_{0}\right)$ of the solid surface (obtained with the help of a shaker for example), the forces $Q_{r n} I_{2} I_{6}$ and $\Delta P_{r n} I_{2} I_{6}$ appearing in equations (17) and (18) are equal to $F_{0} \theta_{r}\left(x_{0}\right) \psi_{n}\left(x_{0}\right)$ and 0 respectively. If the plate is excited acoustically by a plane wave of amplitude $P_{i}$, the pressure applied on the fluid-solid mixture and the pressure difference in the fluid are uniformly distributed. In this case,

$$
Q_{r n}=16 P_{i} / \pi^{2} r n
$$

with $(r, n)$ odd [3]. From reference [11], the values taken for $\Delta P_{r n}$ are $\phi Q_{r n}$.

From previous theoretical works, different mechanisms of energy dissipation can easily be included in the calculations. Forces of viscous frictions in the pores can be introduced in equations $(1,2)$ and $(8,9)$. These forces are derived from a dissipation potential and their effect is accounted for by considering the tortuosity to be complex and frequency dependent,

$$
\tau(\omega)=\tau_{\infty}-\mathrm{j} \frac{\eta \phi}{\kappa \rho_{f} \omega} F(\omega) .
$$

where $\eta$ is the fluid dynamic viscosity and $\kappa$ is the solid permeability. The function $F(\omega)$ is the viscosity correction function and can be evaluated from a model [12]. This function is close to 1 in the low-frequency domain where this study is carried out. The dynamic tortuosity $\tau(\omega)$ is inserted instead of $\tau_{\infty}$ into equations (9) and (18). Structural damping is included in the model by considering complex elastic moduli. For air-saturated materials, the energy loss by thermal exchanges between the solid structure and the fluid can be included by considering a dynamic compressibility [13]. The fluid compressibility appears in Biot's elastic coefficients.

\section{A NOTE ON BIOT'S ELASTIC COEFFICIENTS}

Biot's coefficients of interest in this study are $\alpha$ and $M$ and are given by [6]

$$
\alpha=\phi(1+Q / R), \quad M=R / \phi^{2},
$$

where the elastic coefficients $Q$ and $R$ can be written as

$$
Q=\frac{(1-c) \phi_{s} \phi}{(1-c) \phi_{s} / K_{s}+\phi / K_{f}}, \quad R=\frac{\phi^{2}}{(1-c) \phi_{s} / K_{s}+\phi / K_{f}}
$$

with

$$
c=K / \phi_{s} K_{s} \text { and } \quad \phi_{s}=1-\phi .
$$

In these expressions, $K_{s}, K_{f}$ and $K$ are, respectively, the bulk moduli of the solid constituting the frame, of the fluid and of the frame at constant fluid pressure. The 
coefficient $c$ can be seen as a coefficient characterizing the consolidation state of the heterogeneous material. Since $K$ is less than the Hashin-Strikman upper bound $\phi_{s} K_{s}[14]$, $c$ is between 0 (non-consolidated materials) and 1 (consolidated materials). The following relation is further obtained:

$$
\alpha=1-c \phi_{s}
$$

and the bounds for $\alpha$ are $\phi$ (consolidated materials) and 1 (non-consolidated materials). It is interesting to note the similarity for $c=0$ between the expression for $Q$ and $R$ and the Hashin-Strikman lower bound.

Biot's elastic coefficients can be determined from hydrostatic experiments [15]. Since the fluid is air, the approximation $K_{f} \ll K_{s}, K$ can be made. The materials are fairly rigid and so we shall also consider that $c=1$ and $\alpha=\phi$ in the numerical computation. As a result, the coefficients of interest are approximated by

$$
Q \simeq 0, \quad R \simeq \phi K_{f}, \quad M \simeq K_{f} / \phi
$$

It may be noticed that the flexural rigidity or bending stiffness

$$
D=\frac{E h^{3}}{12\left(1-v^{2}\right)}=\frac{K(1-2 v) h^{3}}{4\left(1-v^{2}\right)}
$$

is a key parameter in the problems of plate vibration. It involves Young's modulus $E$ and the Poisson ratio $v$ of the solid frame. The coefficient $K$ given as a function of $E$ and $v$ by $K=E / 3(1-2 v)$ must therefore be evaluated in any case. As a consequence, the parameters $c, \alpha$ and $M$ can be determined without making approximations if the bulk modulus $K_{s}$ of the solid constituting the porous frame is known.

\section{NUMERICAL AND EXPERIMENTAL RESULTS}

\subsection{PRELIMINARY TESTS}

Three plates were studied. Their properties are summarized in Table 1 . The plates were obtained by consolidating the particles of a material with a binder. The Coustone ${ }^{\dagger}$ plate contains flint particles with a mean grain size of about $1 \mathrm{~mm}$ and an epoxy rubber binder. The other two plates designated as G foam and Y foam are fabricated from particles of plastic foam obtained from recycled car dashboards. Their grain size varies from a few tens of $\mu \mathrm{m}$ to $5 \mathrm{~mm}$ and the binder is manufactured by Hyperlast LTD. ${ }^{\ddagger}$ For each material, the permeability was measured from flow resistivity tests. The tortuosity has not been measured but estimated from Berryman's formula $[16] \tau_{\infty}=(1+1 / \phi) / 2$ as a value between $1 \cdot 2$ and 1.8 depending on the porosity. It was noticed that this coefficient does not have a strong influence on the response of the plates studied. The numerical value of Young's modulus used for the Coustone is the one provided by the manufacturer. For the dashboard foams, $E$ was evaluated experimentally from a simple experiment of bending of a cantilever beam submitted to a known force at the free end and from the corresponding deflection formula.

\footnotetext{
${ }^{\dagger}$ CEP Acoustics Ltd, Unit C Falcon Court, Clayton Business Park, Clayton Le Moors, Accrington BB5 5JD, U.K.

${ }^{\ddagger}$ Hyperlast Ltd, Station Road, Birch Vale, High Peak SK22 1BR, U.K.
} 
TABLE 1

Information necessary for the computation of the plate response

\begin{tabular}{lccc}
\hline & G foam & Y foam & Coustone \\
\hline Lateral size, $a(\mathrm{~m})$ & $0 \cdot 5$ & $0 \cdot 5$ & $0 \cdot 5$ \\
Lateral size, $b(\mathrm{~m})$ & $0 \cdot 5$ & $0 \cdot 5$ & $0 \cdot 5$ \\
Thickness, $h(\mathrm{~mm})$ & 11 & $10 \cdot 70$ & $11 \cdot 5$ \\
Density, $\rho\left(\mathrm{kg} / \mathrm{m}^{3}\right)$ & 348 & 353 & 1295 \\
Young's modulus, $E(\mathrm{~Pa})$ & $4 \cdot 0 \times 10^{6}(1+0 \cdot 15 \mathrm{j})$ & $2 \cdot 1 \times 10^{7}(1+0 \cdot 1 \mathrm{j})$ & $3 \cdot 4 \times 10^{8}(1+0 \cdot 15 \mathrm{j})$ \\
The Poisson ratio, $v$ & $0 \cdot 35$ & $0 \cdot 35$ & $0 \cdot 35$ \\
Porosity, $\phi$ & $0 \cdot 74$ & $0 \cdot 69$ & $0 \cdot 36$ \\
Tortuosity, $\tau_{\infty}$ & $1 \cdot 2$ & $1 \cdot 2$ & $1 \cdot 8$ \\
Permeability, $\kappa\left(\mathrm{m}^{2}\right)$ & $7 \cdot 0 \times 10^{-10}$ & $2 \cdot 7 \times 10^{-10}$ & $4 \cdot 3 \times 10^{-10}$ \\
\hline
\end{tabular}

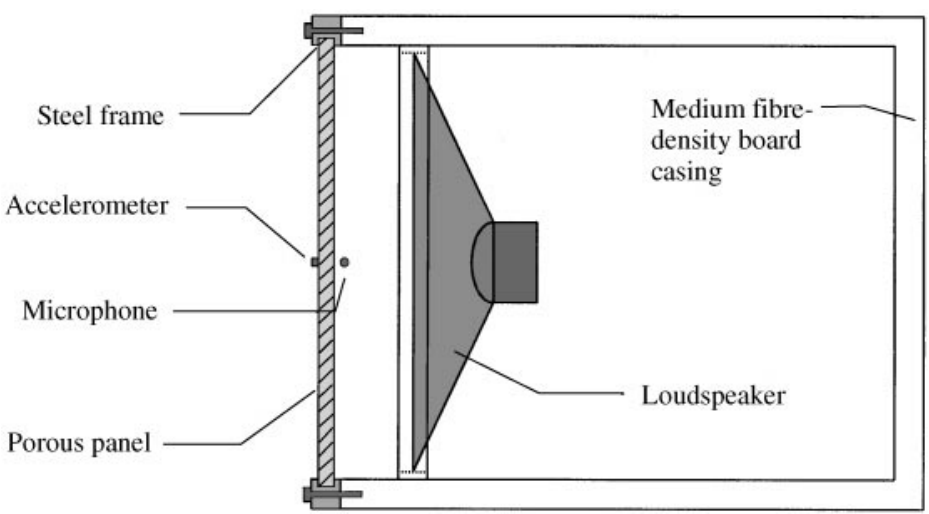

Figure 1. Experimental set-up.

\subsection{NUMERICAL RESULTS}

The plate response to a uniform acoustical excitation was calculated from the variational method described in section 3. The Gauss integration method with 40 terms of Legendre polynomial was used in order to obtain a fast and accurate evaluation of the integrals $\left(I_{1}\right)-\left(I_{6}\right)$. The response was calculated at the centre for three plates. The values for the density and the dynamic viscosity of air are $\rho_{f}=1.2 \mathrm{~kg} / \mathrm{m}^{3}$ and $\eta=1.839 \times 10^{-5} \mathrm{~kg} / \mathrm{m} / \mathrm{s}$ respectively. A typical value of 0.35 is taken for the Poisson ratio for all materials. In a first step, the computation included only the energy loss by viscous friction between the solid and the fluid. From the experimental results, it appeared that the observed damping could not be explained by this mechanism alone. The viscoelastic damping in the solid structure plays an important role and complex Young's modulus and the Poisson ratio had to be considered. An imaginary part between 10 and $15 \%$ of the real part has been taken for $E$ and for $v$ (Table 1). Since a detailed analysis of structural damping is not the subject of this article and for the sake of simplicity, these values were chosen arbitrarily and independent of frequency, in order to match the experimental results on the damping.

\subsection{EXPERIMENTS AND COMPARISON}

The experimental set-up is shown in Figure 1. A porous panel clamped at the four edges was excited by the low-frequency noise produced by a loudspeaker of $40 \mathrm{~cm}$ diameter. 


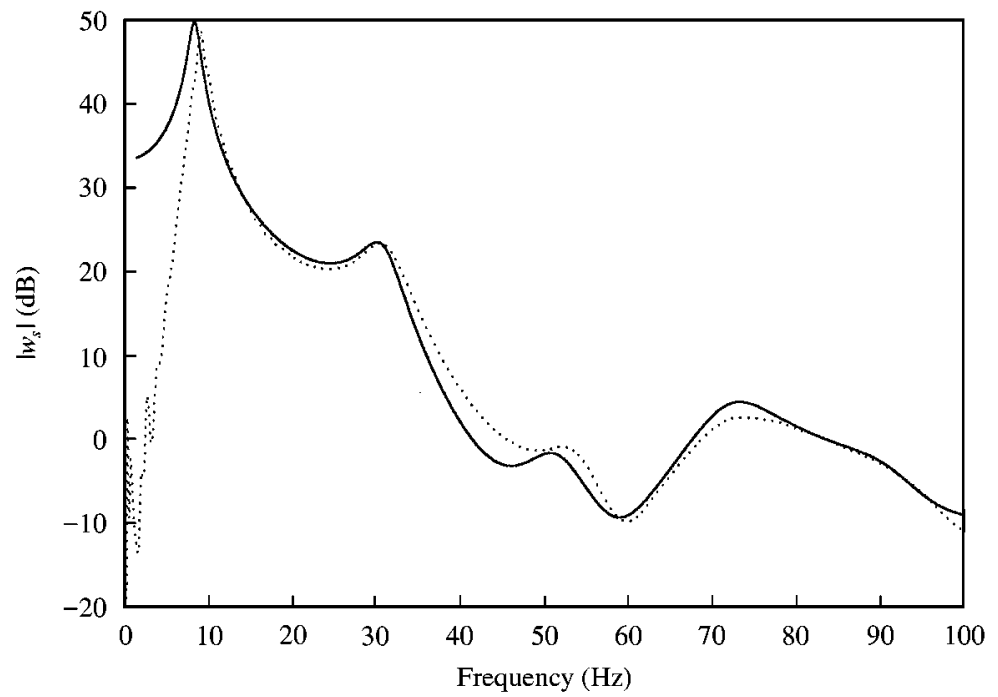

Figure 2. Calculated and measured deflection of the $\mathrm{G}$ foam: $\_$, theory; ……, experiment.

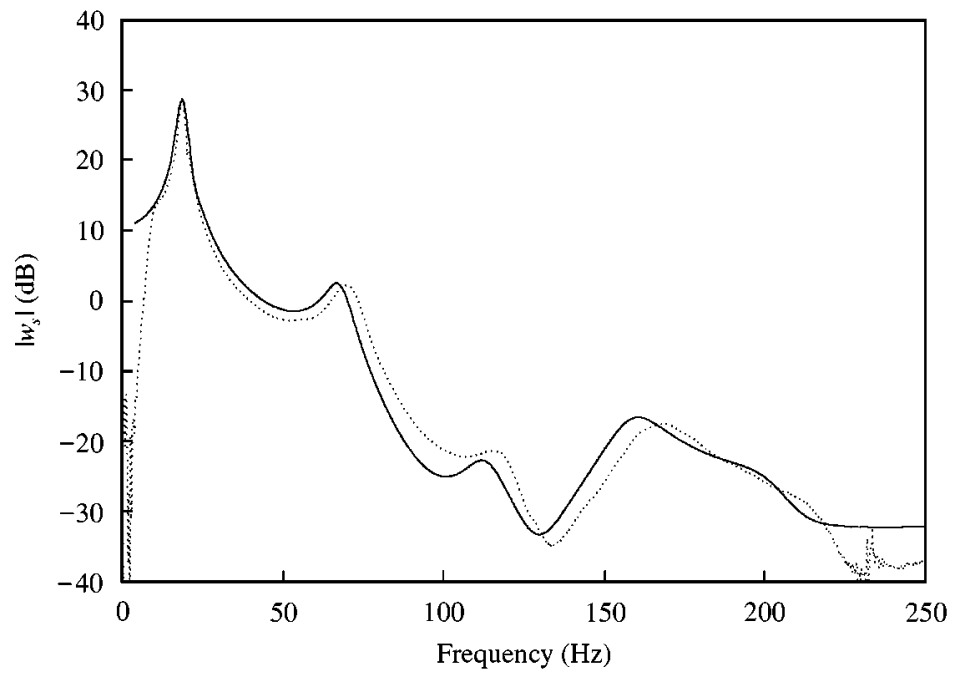

Figure 3. Calculated and measured deflection of the Y foam: __, theory; ……, experiment.

Effective clamping was achieved by using two heavy steel frames of about $25 \mathrm{~kg}$ each such that the mass of the sample was only a few per cent of the mass of the frames. The plate deflection was measured by using an accelerometer of negligible mass (a few grams). With the help of a microphone at $1 \mathrm{~cm}$ from the surface, the amplitude of the incident pressure $P_{i}$ at $(x=a / 2, y=b / 2)$ was evaluated to a value of about $0 \cdot 1 \mathrm{~Pa}$. The theoretical and experimental plate deflections are plotted in Figures 2-4 versus frequency for three different porous plates. Since the absolute value of the experimental deflection was not available, an arbitrary reference value was taken for the theoretical calculation chosen to enable the comparison. The experimental response was measured at $(x=a / 2, y=b / 2)$. Since the plate is excited by a uniform pressure, only the odd modes with an even number of nodal lines are 


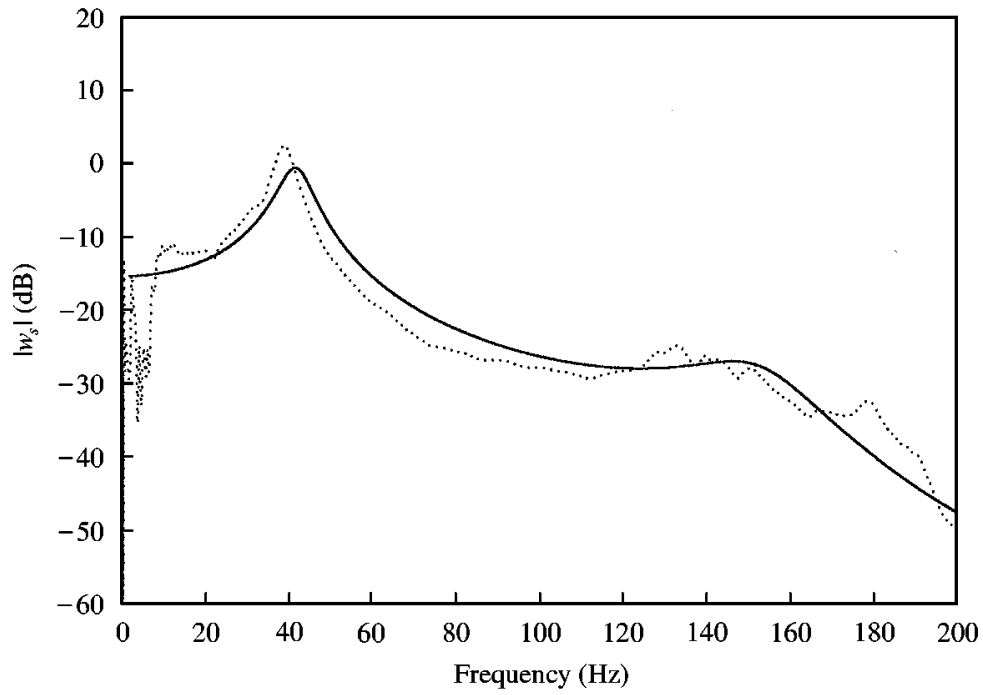

Figure 4. Calculated and measured deflection of the Coustone: ——, theory; ……, experiment.

TABLe 2

First theoretical and experimental resonance frequencies (in $\mathrm{Hz}$ ) for the $\mathrm{G}$ foam plate

\begin{tabular}{lcccc}
\hline Theory & $8 \cdot 5$ & $30 \cdot 3$ & $50 \cdot 8$ & $73 \cdot 1$ \\
\hline Experiment & $\simeq 9$ & $30 \cdot 5$ & $52 \cdot 7$ & $73 \cdot 8$ \\
\hline
\end{tabular}

TABLE 3

First theoretical and experimental resonance frequencies (in $\mathrm{Hz}$ ) for the $\mathrm{Y}$ foam plate

\begin{tabular}{lcccc}
\hline Theory & $18 \cdot 5$ & $66 \cdot 9$ & $111 \cdot 5$ & $159 \cdot 7$ \\
\hline Experiment & $18 \cdot 8$ & $69 \cdot 8$ & 115 & 168 \\
\hline
\end{tabular}

TABLE 4

First theoretical and experimental resonance frequencies (in $\mathrm{Hz}$ ) for the Coustone plate

\begin{tabular}{lcc}
\hline Theory & $41 \cdot 7$ & $147 \cdot 0$ \\
\hline Experiment & 38.8 & $\simeq 133$ \\
\hline
\end{tabular}

excited. A reasonable good agreement is found when comparing the experimental and theoretical frequencies for these modes (see Tables 2-4), with a maximum error around $10 \%$ in the range of samples studied. The resonance frequencies are strongly dependent on Young's modulus $E$, the Poisson ratio $v$ and the porosity. In the case of the G foam, the first resonance, located at about $10 \mathrm{~Hz}$, was in the low-frequency limit sensitivity of the microphone so that the experimental results are not reliable below this frequency. 
The general shape of the experimental curves compares well with the theoretical curves. The discrepancies can be due to the fact that frequency-independent elastic moduli have been considered in the model in this simple analysis. In addition, it has been assumed for the calculations that the incident pressure field was uniformly distributed over the surface of the plate in the whole frequency range, while it is not perfectly constant in the real experimental configuration.

\section{CONCLUSION}

The deflection of a porous plate and the relative fluid-solid displacement are important in the study of the effect of resonances on the surface impedance of porous layers. In this article, a variational method for solving the plate equations has been used and the solutions have been given for different porous materials with the four edges clamped. The effect of fluid loading has been neglected and no external force other than the excitation terms has been taken into consideration. This effect is closely related to the calculation of global vibroacoustic indicators: i.e., the mean square velocity, the radiated sound power and radiation efficiency. Nevertheless, the first comparison between experimental and theoretical results on the plate defection is reasonably good if the viscoelastic damping in the solid is accounted for.

\section{ACKNOWLEDGMENTS}

We are grateful to Prof. A. Cummings for his encouragement and his comment on this article and to E. Chadwick for producing coustone plates. This work has been carried out with the support of the British Engineering and Physical Sciences Research Council (EPSRC) grant number GR/L54905.

\section{REFERENCES}

1. D. D. Theodorakopoulos and D. E. Beskos 1994 Acta Mechanica 103, 191-203. Flexural vibration of poroelastic plates.

2. M. A. BIOT 1956 Journal of the Acoustical Society of America 28, 168-191. Theory of elastic wave propagation in a fluid-saturated porous solid. Parts I and II.

3. R. SzILARD 1974 Theory and Analysis of Plates: Classical ad Numerical Methods. Englewood Cliffs, NJ: Prentice-Hall.

4. M. A. Biot 1962 Journal of Applied Physics 33, 1482-1498. Mechanics of deformation and acoustics propagation in porous media.

5. P. Leclaire, K. V. Horoshenkov and A. Cummings 2001 Journal of Sound and Vibration 247, 1-18. Transverse vibrations of a thin rectangular porous plate saturated by a fluid.

6. M. A. Biot and D. G. Willis 1957 Journal of Applied Mechanics 24, 594-601. The elastic coefficients of the theory of consolidation.

7. D. Young 1950 Journal of Applied Mechanics 17, 448-453. Vibration of rectangular plates by the Ritz method.

8. G. B. WARBURton 1954 Proceedings of the Institution of Mechanical Engineering 168, 371-384. The vibration of rectangular plates.

9. Z. DiNG 1996 Journal of Sound and Vibration 189, 81-87. Natural frequencies of rectangular plates using a set of static beam function in Rayleigh-Ritz method.

10. H. Nelisse, O. Beslin and J. Nicolas 1998 Journal of Sound and Vibration 211, 207-225. A generalized approach for the acoustic radiation from a baffled or unbaffled plate with arbitrary boundary conditions, immersed in a light or heavy fluid.

11. H. Deresiewicz and R. Skalak 1963 Bulletin of the Seismological Society of America 53, 783-788. On uniqueness in dynamic poroelasticity. 
12. J. F. Allard 1993 Sound Propagation in Porous Media: Modelling Sound Absorbing Materials. London: Elsevier.

13. D. Lafarge, P. Lemarinier, J. F. Allard and V. TARnow 1997 Journal of the Acoustical Society of America 102, 1995-2006. Dynamic compressibility of air in porous structures at audible frequencies.

14. Z. Hashin and S. Strikman 1963 Journal of Mechanical Physics and Solids 11, 127-140. A variational approach to the theory of the elastic behaviour of multiphase materials.

15. I. FATT 1959 Journal of Applied Mechanics 26, 296-297. The Biot-Willis elastic coefficients for a sandstone.

16. J. G. Berryman 1980 Applied Physics Letters 37, 382-384. Confirmation of Biot's theory. 